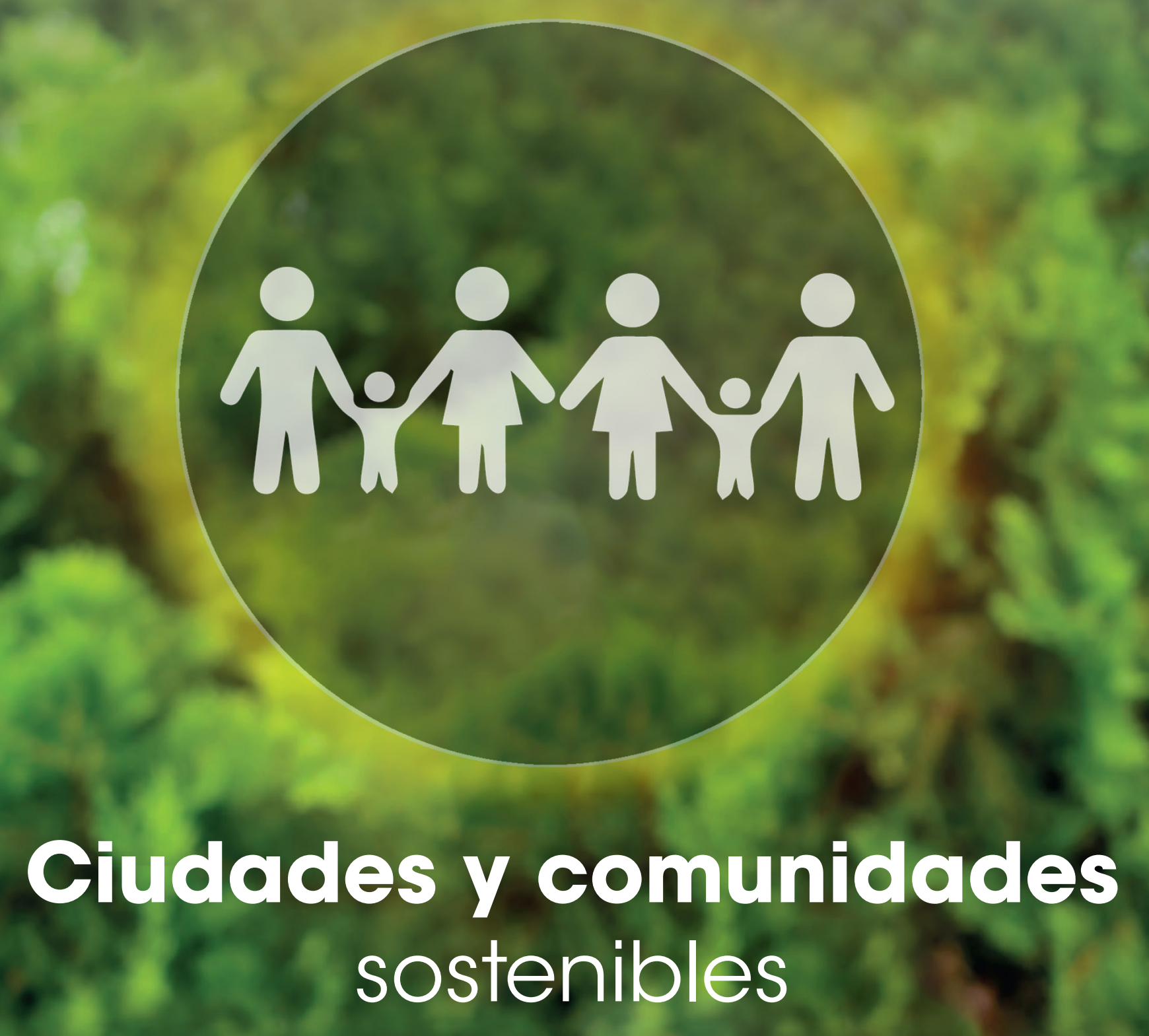




\section{EVALUACIÓN DE LOS FACTORES DE RIESGO \\ EN PAIME - CUNDINAMARCA, PARA EL \\ FORTALECIMIENTO DE ESTE COMPONENTE EN \\ SU ESQUEMA DE ORDENAMIENTO TERRITORIAL}

Julissa C. Méndez Y.

Laura T. Garzón P.1

Universidad de La Salle

\section{Resumen:}

En Paime - Cundinamarca se han presentado eventos adversos generando impactos sociales, económicos y ambientales; esto debido a que no existe percepción del riesgo por la debilidad del componente frente a la realidad del municipio. Por ello es de vital importancia la identificación y evaluación de riesgos dentro del municipio para así fortalecer el Esquema de Ordenamiento Territorial (EOT) que contará con los instrumentos necesarios para la reducción del mismo y la mejora de la capacidad de resiliencia de la población; así, la alcaldía municipal podrá implementar los programas que sean necesarios para preservar la integridad de sus habitantes y su desarrollo sostenible. El proyecto de investigación, tiene como objetivo evaluar las amenazasy el nivel de vulnerabilidad que frente a ellas se presentan en Paime, con el fin de formular alternativas para el fortalecimiento del componente gestión del riesgo en su EOT. Para tal fin, se propone un análisis de la situación real del municipio teniendo en cuenta las condiciones político-administrativas, físicas, económicas y ambientales para evaluar los factores de riesgo a los que se encuentra expuesto y de esta forma poder llegar a la formulación de alternativas para la reducción del riesgo.

Palabras clave: modelo; unidades; formalización, recursos; educación. 


\begin{abstract}
:
In Paime - Cundinamarca there have presented adverse events generating social, economic and environmental impacts; this is because there is no perception of the risk due to the weakness of the component compared to the reality of the municipality. Therefore, it is vitally important to identify and evaluate risks within the municipality in order to strengthen the Esquema de Ordenamiento Territorial (EOT), which will have the necessary instruments to reduce it and improve the resilience of the population; thus, the municipal mayoralty may implement the necessary programs to preserve the integrity of its inhabitants and their sustainable development. The objective of the research project is to evaluate the threats and the level of vulnerability presented in Paime, in order to formulate alternatives for strengthening the risk management component of their EOT. For this purpose, an analysis of the real situation of the municipality is proposed taking into account the political-administrative, physical, economic and environmental conditions to evaluate the risk factors to which it is exposed and thus be able to arrive at the formulation of alternatives for risk reduction.
\end{abstract}

Keywords: Risk management; disaster; vulnerability; threat; territorial planning.

\title{
Introducción
}

La gestión del riesgo es un proceso que se encuentra ligado al desarrollo territorial teniendo como fundamento la formulación, ejecución, seguimiento y evaluación de políticas, estrategias, planes, programas, medidas y acciones que conllevan al conocimiento y la reducción del riesgo y para el manejo de desastres, con el propósito específico de contribuir a la seguridad y el bienestar de las personas y contribuyen al desarrollo sostenible tal como lo contempla la Ley 1523 de 2012 (Pineda Ruíz, Rodríguez Bolaños, \& Barrios Jiménez, 2013). Lo anterior, se fomenta bajo estructuras organizativas, instrumentos de 
planificación, sistemas de información y mecanismos de financiación que hacen posible llevar a cabo el proceso de identificación de las amenazas y vulnerabilidades para concretar acciones que disminuyan la probabilidad del riesgo.

Adicionalmente, la Ley 388 de 1997, suministra orientaciones que posibilita el desarrollo y ordenamiento territorial de un municipio o distrito, teniendo en cuenta la gestión del riesgo de desastres como un objetivo primordial donde la protección del bien público y la calidad de vida de los habitantes tiene una visión preventiva hacia cualquier amenaza que pueda afectar la integridad social y la sostenibilidad. Igualmente, se resalta el requisito de conservación y sostenibilidad ecológica como parte del ordenamiento territorial a fin de disminuir los posibles impactos ambientales negativos que generan una mayor vulnerabilidad en el territorio implicado. Es así como se incluye la sostenibilidad como una directriz que permite el desarrollo de un territorio sin implicar el aumento de los factores de riesgos de desastres (Calderón Ramírez \& Frey, 2017).

Por otro lado, tal como lo indica el Banco Mundial en 2014, se deben tener áreas básicas para fortalecer la capacidad de adaptación frente a diferentes riesgos. Entre estas áreas se encuentran: la identificación de los riesgos, la cual implica comprender los peligros y anticiparse a posibles efectos siendo una herramienta para tomar decisiones; la reducción de los riesgos, teniendo como base información sobre peligros presentes para desarrollar estrategias, planes y proyectos que pueden disminuir el riesgo; preparación, llevándose a cabo a través de sistemas de alerta temprana; protección financiera, siendo una estrategia que salvaguarda a gobiernos, empresas y hogares sobre la carga económica que implica una catástrofe; por último, la reconstrucción que tiene en cuenta la resiliencia. Esta última, representa oportunidades para promover la gestión del riesgo mediante la planificación integrada de la recuperación y restauración que generen una mayor capacidad de adaptación. 
En este orden de ideas, el presente artículo busca describir la identificación de los riesgos asociados al municipio de Paime, que permitirán fortalecer el EOT del mismo, esto a partir de la formulación de alternativas que mejorarán la capacidad de resiliencia de la población. Lo anterior, por medio de la información disponible en bases de datos institucionales y encuestas de percepción de la población, con el objetivo de que la misma comunidad conozca las amenazas a las que están expuestos y el nivel de vulnerabilidad que presentan.

\section{Marco teórico}

El riesgo se puede entender como el resultado de relacionar las amenazas o probabilidades de ocurrencia de un evento y la vulnerabilidad de todo aquello que se encuentra expuesto, donde los sucesos que tienen su origen en la dinámica de la naturaleza no son siempre controlables pero la vulnerabilidad que acompaña a la población y sus bienes sí; de este modo el riesgo se puede clasificar en tres grupos:

1. Riesgos cotidianos o permanentes, asociados a la funcionalidad del territorio

2. Riesgos cíclicos o recurrentes, asociados a las condiciones climáticas

3. Riesgos de baja ocurrencia y alto impacto, asociados a sismos o eventos tecnológicos de media o baja ocurrencia.

\section{Factores de riesgo}

\section{La amenaza.}

La amenaza corresponde a un fenómeno de origen natural, socio-natural y/o tecnológico, en general, definido por su naturaleza, ubicación, recurrencia, probabilidad de ocurrencia, magnitud e intensidad (UNISDR, 2009). En otras palabras, se puede definir como la probabilidad de que ocurra algún evento que por 
sus características pudiera ser catastrófico en un lugar determinado; las amenazas se pueden clasificar en:

a. De origen natural, asociadas a un peligro latente con la posible manifestación de un fenómeno físico cuya génesis se encuentra en los procesos naturales de transformación y modificación de la tierra y el ambiente, estas se suelen clasificar según su origen en tres grupos denominados como: terrestres, atmosféricos y biológicos.

b. De origen socio natural.

c. De origen antrópico (social o tecnológico).

\section{La vulnerabilidad.}

La vulnerabilidad se encuentra íntimamente ligada a los procesos sociales que se desarrollan en áreas que se encuentran en riesgo y tiene que ver

Figura 1. Ciclo de reducción de los factores de vulnerabilidad.

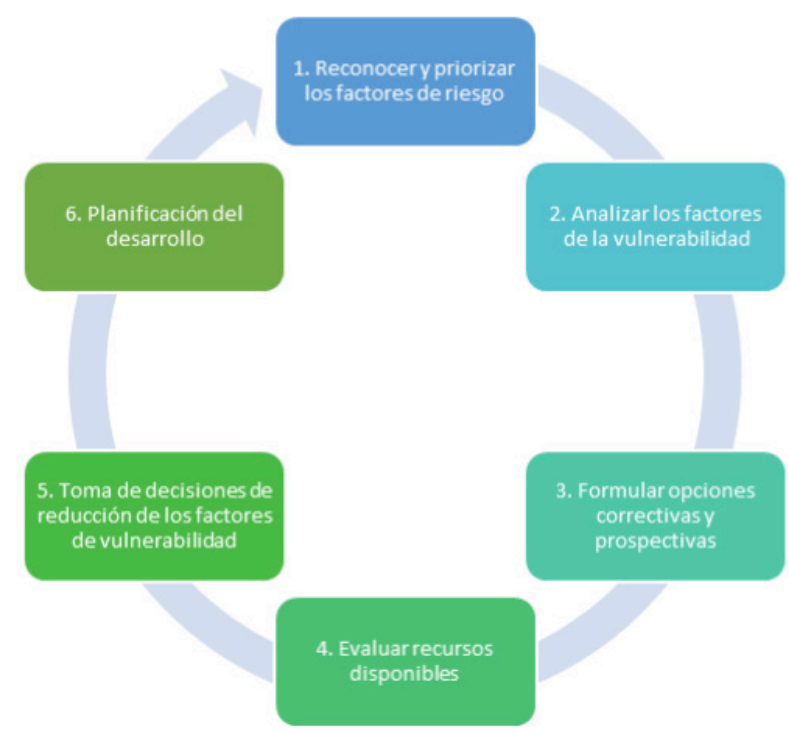

Fuente: Gestión del riesgo. Obtenido de Oficina de Naciones Unidas para la Reducción del Riesgo de Desastres: https://www.eird.org/cd/toolkit08/material/proteccion-infraestructura/gestion_de_riesgo_de_amenaza/8_gestion_de_riesgo.pdf 
directamente con el grado de exposición y la susceptibilidad física, económica, social, ambiental e institucional o la falta de resiliencia de la población ante los diferentes tipos de amenaza. A continuación, se presenta un ciclo de acciones para la reducción de los factores de vulnerabilidad

\section{El ordenamiento territorial.}

Es un instrumento técnico y normativo que busca ordenar el territorio municipal o distrital, el cual debe estar comprendido por un conjunto de objetivos, directrices, políticas, metas, estrategias, programas y actuaciones que permitan orientar y administrar el desarrollo físico del territorio y asimismo la utilización del suelo. Por tanto, según el decreto 879 de 1998, tienen que ser el resultado de la participación constante y efectiva de diferentes actores sociales.

Teniendo en cuenta lo expuesto en el mencionado decreto, los planes de ordenamiento territorial deberán contemplar tres componentes:

1. El componente general, constituido por objetivos, estrategias y contenidos estructurales de largo plazo.

2. El componente urbano, constituido por las políticas, acciones, programas y normas para encauzar y administrar el desarrollo físico urbano.

3. El componente rural, el cual estará constituido por las políticas, acciones, programas y normas para orientar y garantizar la adecuada interacción entre los asentamientos rurales y la cabecera municipal, así como la conveniente utilización del suelo.

Así, el EOT de Paime, cuenta con un conjunto de acciones administrativas y de planificación que orientan el desarrollo del territorio y regulan la utilización, transformación y ocupación del espacio bajo estrategias de desarrollo socioeconómico, sociocultural y en armonía con el medio ambiente. De tal forma, que se orienta una distribución espacial de actividades en función de 
la vocación y aptitud de los suelos, se reduzca la vulnerabilidad ambiental del municipio y concretar lineamiento para la ocupación del territorio teniendo como base estudios de vulnerabilidad, riesgos y amenazas existentes en el municipio (Acuerdo No 004, 2001).

Paime se encuentra localizado en la provincia de Ríonegro, distribuido en cuatro (4) inspecciones y 36 veredas. Limita al oeste con los municipios de Yacopí y Topaipí, al sur con Villagómez, al este con San Cayetano y al norte con los municipios de Muzo, y Cooper en Boyacá. Cuenta con un área de 174,07 km2, representado el $7.4 \%$ del área de la provincia. Paime se encuentra a 151 km de Bogotá D.C., saliendo por la autopista Norte (Alcaldía Municipal de Paime,

Figura 2. Ubicación de Paime

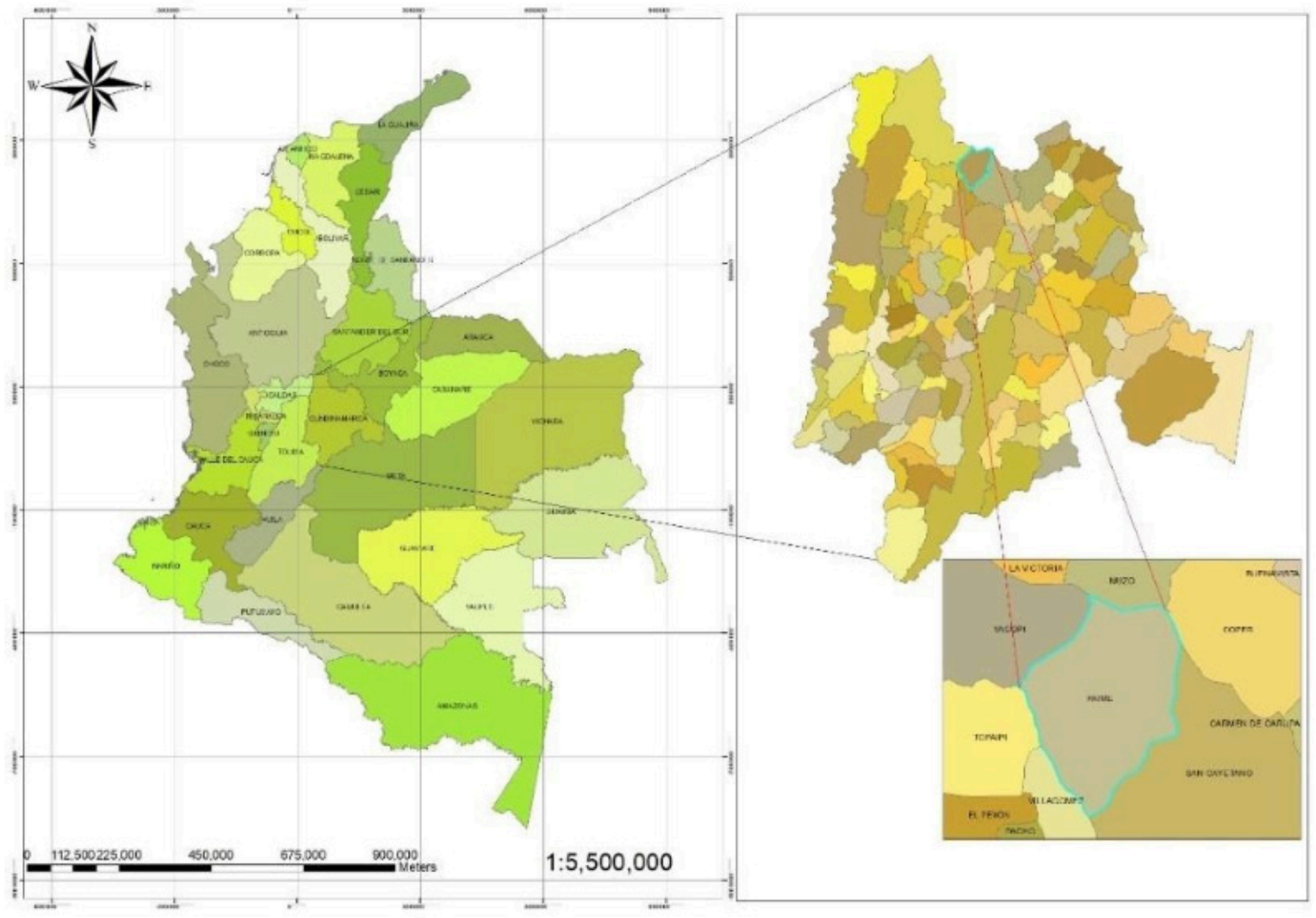

Fuente: elaboración propia. 
2018). Los cuerpos de agua que nacen y atraviesan el municipio pertenecen a la cuenca del Río Minero, teniendo como subcuencas el río Mencipá el cual lo atraviesa por el costado noroccidental siendo fuente de acueductos veredales y el río Negro que cubre a el municipio por el costado nororiental entre los límites con el departamento de Boyacá (Acuerdo No 004, 2001).

\section{Marco legal.}

A continuación, se presenta una relación entre las distintas leyes, decretos y demás normas jurídicas que reglamentan la gestión del riesgo y el ordenamiento del territorio a nivel nacional y municipal.

Tabla 1. Marco legal

\begin{tabular}{|c|c|c|c|}
\hline \multicolumn{2}{|c|}{ NORMA } & ARTÍCULOS Y/O DESCRPCIÓN & APLICACIÓN \\
\hline $\begin{array}{l}\text { Norma de } \\
\text { normas }\end{array}$ & $\begin{array}{l}\text { Constitución Polí- } \\
\text { tica de Colombia } \\
\text { de } 1991\end{array}$ & $\begin{array}{l}\text { Artículo 2: "son fines esenciales del Estado: servir } \\
\text { a la comunidad, promover la prosperidad general } \\
\text { y garantizar la efectividad de los principios, dere- } \\
\text { chos y deberes consagrados en la Constitución; } \\
\text { facilitar la participación de todos en las decisiones } \\
\text { que los afectan y en la vida económica, política, } \\
\text { administrativa y cultural de la Nación; defender la } \\
\text { independencia nacional, mantener la integridad } \\
\text { territorial y asegurar la convivencia pacífica y la } \\
\text { vigencia de un orden justo. } \\
\text { Las autoridades de la República están instituidas } \\
\text { para proteger a todas las personas residentes en } \\
\text { Colombia, en su vida, honra, bienes, creencias, y } \\
\text { demás derechos y libertades, y para asegurar el } \\
\text { cumplimiento de los deberes sociales del Estado } \\
\text { y de los particulares". }\end{array}$ & $\begin{array}{l}\text { Se toma como refe- } \\
\text { rencia debido a la im- } \\
\text { portancia que tiene } \\
\text { en cuanto al deber } \\
\text { de su cumplimiento, } \\
\text { ya que establece la } \\
\text { Ley sobre la integri- } \\
\text { dad territorial que } \\
\text { enmarca las zonas } \\
\text { vulnerables y asenta- } \\
\text { mientos ubicados en } \\
\text { zonas de amenaza. }\end{array}$ \\
\hline
\end{tabular}




\begin{tabular}{|c|c|c|c|}
\hline \multirow[t]{4}{*}{$\begin{array}{l}\text { En cuanto a } \\
\text { Riesgo }\end{array}$} & Ley 46 de 1998 & $\begin{array}{l}\text { En Colombia el Sistema Nacional para la Preven- } \\
\text { ción y Atención de Desastres [SNPAD], descentra- } \\
\text { lizado e interinstitucional, fue creado y organiza- } \\
\text { do mediante la ley } 46 \text { de noviembre de } 1988 \text { y se } \\
\text { reglamenta en su organización y funcionamiento } \\
\text { mediante el Decreto Ley } 919 \text { de mayo de } 1989 \text {. }\end{array}$ & \multirow{4}{*}{$\begin{array}{l}\text { A partir de estas nor- } \\
\text { mas se busca ampliar } \\
\text { y mejorar la infor- } \\
\text { mación actual del } \\
\text { municipio entorno al } \\
\text { componente Gestión } \\
\text { del Riesgo en el EOT } \\
\text { y dar paso a la formu- } \\
\text { lación de alternativas } \\
\text { que permitan dismi- } \\
\text { nuir las condiciones } \\
\text { de riesgo a las que se } \\
\text { encuentra expuesta } \\
\text { la población. }\end{array}$} \\
\hline & Ley 1523 de 2012 & $\begin{array}{l}\text { Por la cual se adopta la Política Nacional de Ges- } \\
\text { tión del Riesgo de Desastres y se establece el Sis- } \\
\text { tema Nacional de Gestión del Riesgo de Desas- } \\
\text { tres y se dictan otras disposiciones. }\end{array}$ & \\
\hline & $\begin{array}{l}\text { Decreto } 2672 \text { de } \\
2013\end{array}$ & $\begin{array}{l}\text { Por la cual se modifica parcialmente la estructura } \\
\text { de la UNGRD, siendo los artículos: 70,10, 12, 13, } \\
16 \text { y } 25 \text { del Decreto número } 4147 \text { de } 2011 \text { y dero- } \\
\text { ga las disposiciones que le sean contrarias. }\end{array}$ & \\
\hline & $\begin{array}{l}\text { Decreto } 308 \text { de } \\
2016\end{array}$ & $\begin{array}{l}\text { Por medio de la cual se adopta el Plan Nacional } \\
\text { de Gestión de Riesgo de Desastres. }\end{array}$ & \\
\hline $\begin{array}{l}\text { En cuanto a Orde- } \\
\text { namiento Territo- } \\
\text { rial }\end{array}$ & Ley 388 de 1997 & $\begin{array}{l}\text { La cual tiene por objetivos: } \\
\text { - Establecer los mecanismos que permitan al mu- } \\
\text { nicipio, en ejercicio de su autonomía, promover el } \\
\text { ordenamiento de su territorio, el uso equitativo y } \\
\text { racional del suelo, la preservación y defensa del } \\
\text { patrimonio ecológico y cultural localizado en su } \\
\text { ámbito territorial y la prevención de desastres en } \\
\text { asentamientos de alto riesgo, así como la ejecu- } \\
\text { ción de acciones urbanísticas eficientes. } \\
\text { - Garantizar que la utilización del suelo por parte } \\
\text { de sus propietarios se ajuste a la función social } \\
\text { de la propiedad y permita hacer efectivos los de- } \\
\text { rechos constitucionales a la vivienda y a los servi- } \\
\text { cios públicos domiciliarios, y velar por la creación } \\
\text { y la defensa del espacio público, así como por la } \\
\text { protección del medio ambientey la prevención de } \\
\text { desastres. }\end{array}$ & $\begin{array}{l}\text { Se hace énfasis sobre } \\
\text { la importancia del } \\
\text { fortalecimiento en la } \\
\text { componente gestión } \\
\text { del riesgo en los POT, } \\
\text { así como en la mejo- } \\
\text { ra de las condiciones } \\
\text { de seguridad de los } \\
\text { asentamientos hu- } \\
\text { manos relacionadas } \\
\text { con la ocupación del } \\
\text { suelo. Es importante, } \\
\text { además, la atención } \\
\text { que se brinde al re- } \\
\text { curso hídrico desde } \\
\text { el fortalecimiento de } \\
\text { los POMCAS. }\end{array}$ \\
\hline
\end{tabular}




\begin{tabular}{|c|c|c|c|}
\hline & Ley 400 de 1997 & $\begin{array}{l}\text { Esta Ley establece los criterios y requisitos míni- } \\
\text { mos para el diseño, construcción y supervisión } \\
\text { técnica de edificaciones nuevas, así como de } \\
\text { aquellas indispensables para la recuperación de } \\
\text { la comunidad con posterioridad a la ocurrencia } \\
\text { de un sismo. }\end{array}$ & \\
\hline & $\begin{array}{l}\text { Decreto } 879 \text { de } \\
1998\end{array}$ & $\begin{array}{l}\text { Por el cual se reglamentan las disposiciones refe- } \\
\text { rentes al ordenamiento del territorio municipal y } \\
\text { distrital y a los planes de ordenamiento territorial. }\end{array}$ & \\
\hline & $\begin{array}{l}\text { Decreto } 1807 \text { de } \\
2014\end{array}$ & $\begin{array}{l}\text { Por el cual se reglamenta el artículo } 189 \text { del De- } \\
\text { creto Ley } 019 \text { de } 2012 \text { en lo relativo a la incorpo- } \\
\text { ración de la gestión del riesgo en los planes de } \\
\text { ordenamiento territorial. }\end{array}$ & \\
\hline & $\begin{array}{l}\text { Guía técnica para } \\
\text { la Formulación de } \\
\text { los POMCAS }\end{array}$ & $\begin{array}{l}\text { En cuanto a la Gestión del Riesgo busca aplicar } \\
\text { criterios técnicos, procedimientos y metodologías } \\
\text { reconocidas y validadas para la inclusión de la } \\
\text { gestión del riesgo en las diferentes fases previstas } \\
\text { para la formulación de los POMCAS. }\end{array}$ & \\
\hline $\begin{array}{l}\text { De carácter muni- } \\
\text { cipal }\end{array}$ & $\begin{array}{l}\text { Acuerdo muni- } \\
\text { cipal No } 004 \text { de } \\
2001\end{array}$ & $\begin{array}{l}\text { Por el cual se adopta el Esquema de Ordenamien- } \\
\text { to Territorial Municipal, se clasifican y determinan } \\
\text { usos del suelo y se establecen los sistemas es- } \\
\text { tructurantes y planes parciales. }\end{array}$ & $\begin{array}{l}\text { Tiene como fin la } \\
\text { comparación de es- } \\
\text { tos documentos con } \\
\text { las políticas y normas } \\
\text { vigentes teniendo en } \\
\text { cuenta la situación } \\
\text { actual del municipio. }\end{array}$ \\
\hline
\end{tabular}

\section{Materiales y métodos}

El desarrollo del proyecto se lleva a cabo en cuatro fases las cuales posibilitan recolectar la información pertinente y evaluar los factores de riesgo que se 
presentan en Paime. En la primera fase se recopila toda la información disponible en las bases de datos de la Alcaldía Municipal tales como IDEAM ${ }^{5}$, IGAC ${ }^{6}$ , DANE ${ }^{7}$, UNGRD $^{8}$ e SIDHMA ${ }^{9}$; la cual será de relevancia para dar paso a la segunda fase donde se realizará el análisis situacional actual del municipio. Lo anterior, permite establecer los componentes social, económico, político-administrativo, físico, biótico y ambiental, obteniendo respectivamente datos como: demografía, salud, educación e infraestructura; presupuesto municipal y actividades económicas; organización, entidades municipales y normatividad vigente; localización, división política, clima, hidrografía, entre otros; flora y fauna; actividades que generan impactos ambientales.

Adicionalmente, se complementa la información a partir de encuestas que se efectúan a los habitantes y las instituciones para conocer la percepción de quienes habitan el municipio sobre su entorno, sus recursos y las amenazas a las cuales se encuentran expuestos. Por consiguiente, la tercera fase implica la exploración y caracterización de los escenarios de riesgo, donde se identifican y evalúan las amenazas y vulnerabilidades presentes en el municipio que llevan a la condición de riesgo del mismo a partir de las dos fases anteriores. Es de resaltar que estos datos se obtienen por medio de matrices e indicadores que reflejan los riesgos y vulnerabilidades, de tal forma que se ordena la valoración de los impactos sobre cada componente.

Específicamente, se hace uso de la metodología propuesta por López (2016), en la que se establece una escala de calificación de 1 a 10 para la amenaza, como el total de la sumatoria de los aspectos de frecuencia (poco probable -PP-, probable -P-, muy probable -MP-), intensidad (baja, media, alta y muy alta) y cobertura (poca, media, alta); como se muestra en la siguiente tabla:

Instituto de Hidrologia Metereologica y Estudios Ambientales

Instituto Geográfico Agustín Codazzi

'Departamento Administrativo Nacional de Estadística

- Unidad Nacional para la Gestión del Riesgo de Desastres

'Sistema de Información sobre desastres, hidrometeorología y medio ambiente de la Universidad de la Salle 
Tabla 2. Criterios de calificación para las amenazas

\begin{tabular}{|c|c|c|c|c|c|c|c|c|}
\hline \multicolumn{2}{|c|}{ Frecuencia } & \multirow{2}{*}{$\begin{array}{c}\text { Calificación } \\
1\end{array}$} & \multicolumn{2}{|c|}{ Intensidad } & \multirow{2}{*}{$\begin{array}{c}\text { Calificación } \\
1\end{array}$} & \multicolumn{2}{|c|}{ Magnitud O Cobertura } & \multirow{2}{*}{$\begin{array}{c}\text { Calificación } \\
1\end{array}$} \\
\hline$P P$ & Poco probable & & $B$ & Baja & & $P$ & Poca & \\
\hline $\boldsymbol{P}$ & Probable & 2 & $M$ & Media & 2 & $M$ & Media & 2 \\
\hline \multirow{2}{*}{ MP } & \multirow{2}{*}{ Muy Probable } & \multirow{2}{*}{3} & $A$ & Alta & 3 & $A$ & Alta & 3 \\
\hline & & & MA & Muy alta & 4 & Máximo Total & \multicolumn{2}{|r|}{10} \\
\hline
\end{tabular}

Fuente: López, L. (2016). Apuntes de clase, espacio académico Gestión del Riesgo. En Metodologías de evaluación. Bogotá: Programa de Ingeniería Ambiental y Sanitaria. Unisalle.

En cuanto a la vulnerabilidad se calificó en una escala de 0.1 a 1.0 de acuerdo con las condiciones en que se encuentra el municipio y los eventos a los cuales está expuesto, de este modo se tuvo en cuenta lo siguiente:

Tabla 3. Criterios de calificación para la vulnerabilidad.

\begin{tabular}{|c|c|}
\hline VULNERABILIDAD & RANGO \\
\hline BAJA & $0,1-0,3$ \\
\hline MEDIA & $0,4-0,6$ \\
\hline ALTA & $0,7-1.0$ \\
\hline
\end{tabular}

Fuente: López, L. (2016). Apuntes de clase, espacio académico Gestión del Riesgo. En Metodologías de evaluación. Bogotá: Programa de Ingeniería Ambiental y Sanitaria. Unisalle.

Finalmente, para la evaluación del riesgo se tendrá en cuenta que el nivel de Riesgo (R) será igual a la amenaza (A) por la vulnerabilidad $(V)$ [R=A*V], donde se tendrá en cuenta la siguiente tabla: 
Tabla 4. Criterios de calificación para el riesgo.

\begin{tabular}{|c|c|}
\hline Riesgo & Rango \\
\hline Bajo & $1-2,9$ \\
\hline Medio & $3-4,9$ \\
\hline Medio - Alto & $5-6,9$ \\
\hline Alto & $7-8,9$ \\
\hline Muy Alto & $\geq 9$ \\
\hline
\end{tabular}

Fuente: López, L. (2016). Apuntes de clase, espacio académico Gestión del Riesgo. En Metodologías de evaluación. Bogotá: Programa de Ingeniería Ambiental y Sanitaria. Unisalle.

La última fase está relacionada con la formulación de alternativas. Implica una comparación entre el contenido del EOT de Paime y la realidad del municipio encontrada a partir de la información de las fases anteriores. Adicionalmente, se formularán alternativas para cada escenario de riesgos identificados, los cuales integrarán acciones a tener en cuenta para la prevención y disminución del riesgo.

\section{Resultados}

El municipio de Paime cuenta con aproximadamente 5600 habitantes, donde de acuerdo con el censo del Departamento Administrativo Nacional de Estadísticas (DANE) para el año 2005, su población se encontraba conformada en un 47\% por mujeres (minoría) y en un 53\% por hombres (mayoría), donde las edades oscilaban de 0 a 85 años, siendo mayor la población con edades entre 9 y 25 años prevaleciendo las edades entre 65 a 85 años; para el año 2013 se observó una disminución del 1.8\% de la población. 
Figura 3. Población de Paime por sexo

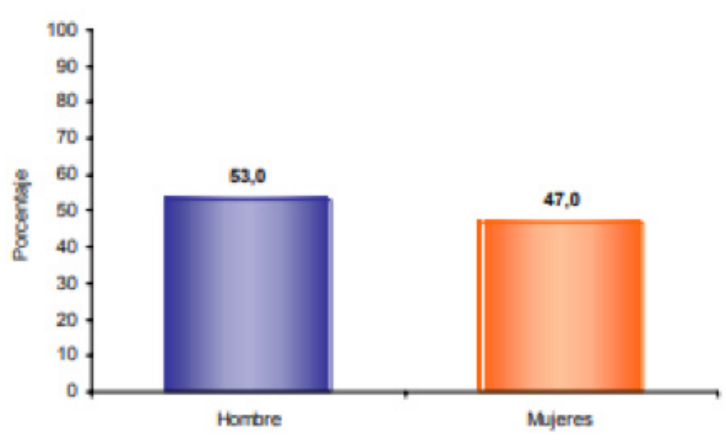

Fuente: DANE, 2006

Para el año 2011, en el plano de diagnóstico situacional del municipio para llevar a cabo la formulación del Plan de Desarrollo Municipal (PDM), se encontró la población conformada en su mayoría por mujeres y las edades con mayor población se encontraban entre los 41 a 65 años.

Tabla 5. Población de Paime por sexo

\begin{tabular}{|c|c|c|c|c|c|c|}
\hline Grupo & $\begin{array}{c}1 \text {-4 } \\
\text { años }\end{array}$ & $\begin{array}{l}5-12 \\
\text { anos }\end{array}$ & $\begin{array}{c}13 \text { - } 18 \\
\text { años }\end{array}$ & $\begin{array}{l}19 \text { - } 25 \\
\text { años }\end{array}$ & $\begin{array}{c}26 \text { - } 40 \\
\text { años }\end{array}$ & $\begin{array}{r}41 \text { - } 65 \\
\text { años }\end{array}$ \\
\hline Mujeres & 224 & 424 & 264 & 325 & 684 & 844 \\
\hline Hombres & 201 & 407 & 252 & 287 & 606 & 806 \\
\hline TOTAL & 425 & 331 & 516 & 612 & 1290 & 1650 \\
\hline
\end{tabular}

Fuente: PDM, 2008 
Los servicios públicos con los que cuenta la población del municipio son acueducto con una cobertura de cerca del $40 \%$ a nivel rural (cuatro veredas y cuatro inspecciones) con grandes deficiencias en sus líneas de conducción y calidad de las aguas; es por ello que la mayoría de la población se abastece del agua de nacederos y quebradas vecinas. Por otro lado, el alcantarillado es deficiente con una cobertura de apenas el 8.3\% sin red a nivel veredal. En cuanto al casco urbano cuenta con un $85 \%$ de cobertura, electricidad prestada por la empresa CODENSA S.A con una cobertura de cerca del 60\% en el área rural con un promedio entre 8 y 10 horas diarias de servicio y el poblado cuenta con cobertura del $100 \%$.

\section{Estructura urbana.}

En su cabecera municipal, Paime posee una extensión de 12 (8950 m2) hectáreas con topografía quebrada donde sus pendientes oscilan entre 5 y 30\% lo cual no permite que sea fácil el desarrollo urbanístico, el territorio en su extensión total del suelo urbano contempla solo 128.000 m2 con suelo en uso distribuidos de la siguiente forma:

Tabla 6. Uso del suelo urbano

\begin{tabular}{|c|c|}
\hline USO & AREA(M2) \\
\hline Vivienda & 70.590 \\
\hline Mixto & 9.760 \\
\hline Sin Desarrollar (agricola) & 48.600 \\
\hline TOTAL & 128.950 \\
\hline
\end{tabular}

Fuente: PDM, 2008

Según el EOT de Paime, gran parte del territorio presenta problemas de geoinestabilidad reflejados en fenómenos de remoción en masa rotacionales y movimientos de reptación, esto principalmente en el sureste y noreste del 
mismo, donde cabe hacer énfasis que se han detectado asentamientos humanos alrededor de estas zonas que se encuentran en situación de riesgo.

Adicional a lo anterior, se encontró un historial (desde 2007) de eventos que afectaron el municipio desencadenando desastres, este se presenta en la tabla a continuación.

Tabla 7. Eventos presentados en Paime desde el año 2007

\begin{tabular}{|c|c|c|c|}
\hline Fecha & Evento & Afectaciòn & Fuente \\
\hline Marzo de 2007 & Inundación & $\begin{array}{l}\text { En las inspecciones de Cuatro Caminos y Tole- } \\
\text { do, se vieron afectadas } 7 \text { familias, } 35 \text { personas, } \\
7 \text { viviendas averiadas y } 1 \text { centro educativo. }\end{array}$ & UNGRD \\
\hline $\begin{array}{l}\text { Diciembre de } \\
2010\end{array}$ & Deslizamiento & $\begin{array}{l}\text { Resultaron afectadas } 330 \text { familias y } 1320 \text { perso- } \\
\text { nas, lo anterior en la vía San Cayetano- Paime y } \\
\text { Paime - Boquerón. }\end{array}$ & UNGRD \\
\hline Marzo de 2011 & Deslizamiento & $\begin{array}{l}\text { Se afectaron } 16 \text { familias de las veredas Santuario } \\
\text { y La Unión, conformadas por } 80 \text { personas, } 2 \text { de } \\
\text { las viviendas resultaron averiadas, además de ello } \\
\text { resulto } 1 \text { vía afectada. }\end{array}$ & UNGRD \\
\hline Abril de 2011 & Deslizamiento & $\begin{array}{l}\text { Resultaron afectadas } 46 \text { familias para un total de } \\
230 \text { personas, asimismo todas las } 46 \text { viviendas } \\
\text { quedaron averiadas. Lo anterior ocurrió en la } \\
\text { zona urbana y rural, en las inspecciones de Cuatro } \\
\text { Caminos y El Plomo. }\end{array}$ & UNGRD \\
\hline Junio de 2011 & Inundación & $\begin{array}{l}\text { Durante este evento resultaron afectadas } 221 \text { fa- } \\
\text { milias, para un total de } 328 \text { personas, es de anotar } \\
\text { que todas las } 221 \text { viviendas quedaron afectadas. }\end{array}$ & UNGRD \\
\hline Junio de 2011 & Vendaval & $\begin{array}{l}74 \text { familias, las cuales resultan un total de } 222 \text { per- } \\
\text { sonas. Adicional a lo anterior, se afectaron } 92 \mathrm{Ha} \\
\text { de territorio rural. }\end{array}$ & UNGRD \\
\hline
\end{tabular}




\begin{tabular}{|c|c|c|c|}
\hline Marzo de 2012 & Deslizamiento & $\begin{array}{l}\text { El evento se presentó en la vereda La Unión, en } \\
\text { esta resultaron afectadas } 30 \text { personas, las cuales } \\
\text { constituyen } 6 \text { familias, de estas } 2 \text { viviendas queda- } \\
\text { ron destruidas y } 4 \text { viviendas averiadas. }\end{array}$ & UNGRD \\
\hline Julio de 2012 & Incendio forestal & $\begin{array}{l}\text { Se generó en la vereda Tau-Tao, con afectación de } \\
3 \text { hectáreas de vegetación nativa. }\end{array}$ & UNGRD \\
\hline Octubre de 2012 & Inundación & $\begin{array}{l}\text { Se afectaron } 196 \text { personas, } 49 \text { familias y } 49 \text { vivien- } \\
\text { das averiadas. }\end{array}$ & UNGRD \\
\hline $\begin{array}{l}\text { Septiembre de } \\
2012\end{array}$ & Vendaval & $\begin{array}{l}\text { Afectadas } 150 \text { personas, } 30 \text { familias, } 30 \text { viviendas } \\
\text { averiadas de las veredas La Unión, El Plomo, Tau- } \\
\text { Tao, Tolunza, El Triunfo, El Palmar y Lusitana. Asi- } \\
\text { mismo, resultaron afectados los centros educati- } \\
\text { vos de las veredas La Unión, El plomo, Lusitana y } \\
\text { El Palmar. }\end{array}$ & UNGRD \\
\hline Julio de 2013 & Vendaval & $\begin{array}{l}\text { Se afectaron } 270 \text { personas lo que corresponde a } \\
56 \text { familias, con todas las } 56 \text { viviendas averiadas. } \\
\text { Adicional a lo anterior se afectó } 1 \text { vía, } 1 \text { centro de } \\
\text { salud, } 1 \text { centro educativo, } 1 \text { iglesia, } 1 \text { polideportivo } \\
\text { y } 6 \text { Ha de cultivos, se presentaron caídas de redes } \\
\text { eléctricas y la vía principal fue afectada por des- } \\
\text { bordamiento de la quebrada La Mina. }\end{array}$ & UNGRD \\
\hline $\begin{array}{l}\text { Septiembre de } \\
\qquad 2014\end{array}$ & Incendio forestal & $\begin{array}{l}\text { El evento se presenta en las veredas San Carlos, } \\
\text { Moturo y La Trucha, la afectación fue de } 20 \text { hec- } \\
\text { táreas de bosque nativo. }\end{array}$ & UNGRD \\
\hline Abril de 2015 & Inundación & $\begin{array}{l}\text { Durante el evento se afectaron } 10 \text { vías, } 1 \text { puente } \\
\text { vehicular y } 3 \text { puentes peatonales. La creciente sú- } \\
\text { bita en el río Blanco provocó pérdida de la banca } \\
\text { en la vía Paime - Pacho - Bogotá, el colapso del } \\
\text { puente vehicular y un muro de contención; adi- } \\
\text { cionalmente, en la vía Paime - Cuatro Caminos se } \\
\text { presentaron deslizamientos que taponaron la vía, } \\
\text { la red vial terciaria que comunica la cabecera mu- } \\
\text { nicipal con las veredas se afectó gravemente. }\end{array}$ & UNGRD \\
\hline
\end{tabular}




\begin{tabular}{|c|c|c|c|}
\hline Abril de 2016 & Deslizamiento & $\begin{array}{l}\text { Se generó un deslizamiento debido a las Iluvias en } \\
\text { la vereda Ticaba, lo que afecto y tapono la vía que } \\
\text { conduce a las veredas Trinidad y La Unión. }\end{array}$ & UNGRD \\
\hline Junio de 2016 & Deslizamiento & $\begin{array}{l}\text { Se vieron afectadas } 5 \text { personas lo que constituye } \\
1 \text { familia, su vivienda (1) quedo averiada, adicional } \\
\text { a lo anterior } 1 \text { vía (hacía Bogotá) se vio afectada, } \\
\text { esto ocurrió en la vereda San Rafael. }\end{array}$ & UNGRD \\
\hline Agosto de 2016 & Vendaval & $\begin{array}{l}\text { Durante el evento se afectaron } 24 \text { personas las } \\
\text { que constituyen } 6 \text { familias, de estas familias sus } 6 \\
\text { viviendas quedaron averiadas. Adicionalmente, se } \\
\text { afectó } 1 \text { centro educativo, esto ocurrió en la ins- } \\
\text { pección de Venecia. }\end{array}$ & UNGRD \\
\hline Abril de 2017 & Deslizamiento & $\begin{array}{l}\text { El evento afectó } 5 \text { personas ( } 1 \text { familia), la vivien- } \\
\text { da (1) quedo destruida esto en la vereda Esmirna, } \\
\text { además, la vía Paime - vereda Esmirna - San Caye- } \\
\text { tano se tapono totalmente debido al evento. }\end{array}$ & UNGRD \\
\hline Agosto de 2017 & Vendaval & $\begin{array}{l}\text { Se afectaron } 185 \text { personas, lo que equivale a } 37 \\
\text { familias, las } 37 \text { viviendas quedaron averiadas. }\end{array}$ & UNGRD \\
\hline Octubre de 2017 & Deslizamiento & $\begin{array}{l}\text { Generó represamiento en la quebrada Matilde o } \\
\text { Luis Pata, lo que afecto un puente vehicular aguas } \\
\text { abajo sobre el Rio Caney, dejando comprometidas } \\
\text { las veredas San Luis, Mercedes y Centro. }\end{array}$ & UNGRD \\
\hline $\begin{array}{l}\text { Diciembre de } \\
2017\end{array}$ & Incendio forestal & $\begin{array}{l}\text { El evento ocurrió en la vereda Capira, quedando } \\
\text { afectadas } 2 \text { hectáreas entre pastizales y rastrojo. }\end{array}$ & UNGRD \\
\hline Agosto de 2018 & Incendio forestal & El evento afectó las veredas Combuco y La Carrera. & Alcaldía de Paime \\
\hline $\begin{array}{c}\text { Septiembre de } \\
2019\end{array}$ & Incendio forestal & $\begin{array}{l}\text { El evento afectó las veredas Combuco, Santa tere- } \\
\text { sa y Tao Tao }\end{array}$ & El Espectador \\
\hline
\end{tabular}




\section{Discusión}

La metodología escogida ha permitido hasta este punto identificar en primer lugar, los principales eventos que se han presentado en los últimos años en el municipio de Paime, destacándose especialmente los deslizamientos, los cuales han afectado a cientos de familias y agricultores de diferentes zonas de este a lo largo del tiempo. Hecho que se debe a los asentamientos humanos que se encuentran ubicados en zonas de difícil acceso y alto riesgo, ya que el municipio se caracteriza por estar entre zonas montañosas de alta pendiente donde pueden oscilar desde el 3\% hasta el 50\%. Por la misma razón, se han visto afectados espacios públicos como las vías de acceso ubicadas en zonas de alto riesgo de deslizamiento, las cuales son taponadas durante los eventos y dejan incomunicados a la comunidad.

A lo anterior, se suma el incremento de la deforestación debido al aumento de actividades agrícolas y pecuarias en áreas que están destinadas a la conservación de bosques; lo cual implica un aumento de la susceptibilidad del suelo por erosión a causa del mal manejo del uso potencial del suelo y el agotamiento de la capacidad nutritiva de los suelos. Adicionalmente, se encuentra otro factor asociado a este evento el cual es la oferta hídrica del municipio, donde se presenta en promedio una precipitación anual de $252.48 \mathrm{~mm}$, siendo las más altas en abril (390,8 mm) y octubre $(383,7 \mathrm{~mm})$, asociándose a la ocurrencia del evento de deslizamiento, tal como se identifica en la tabla 7.

Por otro lado, se generan eventos de inundación los cuales se asocian a los periodos altos de precipitación (abril y octubre) que se presentan en el municipio; principalmente, en zonas o asentamientos cercanos a las riveras de los ríos y quebradas por el desbordamiento de las mismas, aunque con baja probabilidad de ocurrencia debido a la fisiografía del municipio. Aunque cabe destacar que diferentes quebradas y rondas de ríos han sido usados como botaderos lo que incide en un represamiento y aumenta la posibilidad de las inundaciones en zonas habitadas. 


\section{Conclusiones}

- Con base en la revisión del EOT del municipio en su componente gestión del riesgo se determinó que requiere ajustes en cuanto a lo estipulado en la ley 1523 de 2012 y la ley 388 de 1997 (ordenamiento territorial). Esto es necesario para poder garantizar la concordancia y el fortalecimiento del documento técnico; por tanto, es de gran importancia que la oficina de planeación del municipio plantee alternativas mediante un plan de acción que permita fortalecer el componente gestión del riesgo evidenciando el cumplimiento de la normatividad en cuanto a infraestructura.

- A partir de los eventos presentados y la información disponible en el EOT, fue posible determinar que los asentamientos humanos que están en mayor peligro son los localizados en zonas cercanas a fuentes hídricas superficiales y de alta pendiente, afectando las actividades agrícolas y ganaderas que implican una explotación y uso del suelo.

- El municipio al no ser tan reconocido turísticamente cuenta con pocas fuentes de empleo lo cual impide el progreso de los habitantes que prefieren desplazarse a otros lugares con más posibilidades y ofertas laborales dejándolo expuesto en cuanto a la dimensión social por la desintegración y la dimensión política.

- A partir de los datos recopilados, se observa que el evento que mayor amenaza representa para la comunidad de Paime son los deslizamientos o movimientos en masa de reptación y rotacionales; los cuales se presentan principalmente por la topografía del territorio con altas pendientes y adicional a ello la deforestación que ha ido aumentado por la extensión de zonas agrícolas y ganaderas, erosionando el suelo y disminuyendo su concentración de nutrientes. 


\section{Referencias bibliográficas}

Acuerdo \# 004, Por el cual se adopta el Esquema de Ordenamiento Territorial Municipal, se clasifican y determinan usos del suelo y se establecen los sistemas estructurantes y planes parciales (Concejo Municipal Paime, 09 de marzo de 2001).

Alcaldía Municipal de Paime. (2018). Alcaldía Municipio Paime. Obtenido de: http://www. paime-cundinamarca.gov.co/municipio/nuestro-municipio

Banco Mundial. (2014). Gestión del riesgo de desastres para un desarrollo con capacidad de adaptación. Obtenido de https://www.bancomundial.org/es/results/2013/04/12/managing-disaster-risks-resilient-development

Calderón Ramírez, D., \& Frey, K. (2017). El ordenamiento territorial para la gestión del riesgo de desastres en Colombia. Territorios, (36), 239264. Doi: http://dx.doi.org/10.12804/revistas. urosario.edu.co/territorios/a.4795

DANE. (2005). Boletín censo general 2005, Perfil Paime- Cundinamarca. Obtenido de: https://www.dane.gov.co/files/censo2005/perfiles/ cundinamarca/paime.pdf

Estrategia Internacional para la Reducción de Desastres de las Naciones Unidas (UNISDR). (2009). Terminología sobre Reducción del Riesgo de Desastres. Obtenido de: https://www.unisdr.org/files/7817_UNISDRTerminologySpanish.pdf

López, L. (2016). Apuntes de clase, espacio académico Gestión del Riesgo. En Metodologías de evaluación. Bogotá: Programa de Ingeniería Ambiental y Sanitaria. Unisalle.

Malaver, I. (2008). Plan de Desarrollo Municipal de Paime Cundinamarca. Obtenido de: http://cdim.esap.edu.co/BancoMedios/ Documentos\%20PDF/plan\%20de\%20desarrollo\%20paime\%20-\%20cundinamarca\%20 -\%202008\%20-\%202011.pdf

Pineda Ruíz, L., Rodríguez Bolaños, A., \& Barrios Jiménez, L. (2013). La Gestión del Riesgo de Desastres. Un proceso de desarrollo. Obtenido de https://www.cortolima.gov.co/ sites/default/files/images/stories/gestion_ riesgos/Gestion_del_Riesgo/Modulos/Modulo_IV_260913_Alta.pdf

UNDRR. (2008). Gestión del riesgo. Obtenido de Oficina de Naciones Unidas para la Reducción del Riesgo de Desastres: https:// www.eird.org/cd/toolkit08/material/proteccion-infraestructura/gestion_de_riesgo_de_ amenaza/8_gestion_de_riesgo.pdf 